understand relationships between interventions and exposures (or risk factors) and disease or behavioral outcomes. In general, this approach allows many potential risk factors to be recast theoretically as an intervention. For example, a behavior such as condomless sex, which could never be assigned in an RCT, could be thought of as an 'intervention' or perhaps more appropriately, as the comparison to an intervention of sex with a condom. Once we reframe the question, several methods are available to account for potential biases that may arise in observational studies, including selection bias and confounding.

In this session, we will consider this reframing process using examples, real and hypothetical, from the fields of sexually transmitted infections and HIV. The process typically begins with a carefully constructed causal diagram, called a directed acyclic graph or DAG. DAGs are used to outline the causal pathways related to the research question. The DAG accounts for both the specific factors and temporality of the relationships. With the DAGs in hand, we can plan the appropriate analyses for the specific research questions. Without equations or complex statistics, we will consider three commonly used analytical approaches: propensity scores, instrumental variables, and marginal structural models (epidemiology's MSM). We will also briefly consider other quasi-experimental approaches for policy evaluations.

\section{PL14 WHAT'S TO KNOW AND WHAT'S TO BE DONE - IMPLEMENTING AND MONITORING STI AND HIV PROGRAMMES}

Y Pillay*. Clinton Health Access Initiative, Pretoria, South Africa

10.1136/sextrans-2021-sti.14

With decades of experience implementing STI and HIV programmes globally what do we know and what are we still learning? Implementation science is a relatively new approach to understanding what facilitates rapid implementation and the barriers to implementation.

With a rising tide of some STIs and need to eliminate HIV as a public health threat, this presentation will focus on experiences of a national programme manager implementing STI, HIV and other programmes in an upper middle-income country - South Africa. Looking ahead does the COVID-19 pandemic provide us with opportunities to do things differently and if yes, what can they be?

\section{PL15 DEVELOPMENT OF IMMUNOTHERAPEUTIC APPROACHES AGAINST GONORRHEA}

S Ram* , S Gulati, J Shaughnessy, P Rice. University of Massachusetts Medical School, Worcester, USA

10.1136/sextrans-2021-sti.15

Multidrug-resistant (MDR) Neisseria gonorrhoeae $(\mathrm{Ng})$ is a global health problem. Targeting virulence factors can circumvent the ability of gonococci to resist conventional antimicrobials. Resistance to such agents, if it were to occur, would result loss of bacterial fitness; attenuated 'escape mutants' would be susceptible to host immune defenses. A unique gonococcal immune evasion strategy involves capping of lipooligosaccharide (LOS) with sialic acid by gonococcal sialyltransferase (Lst), utilizing host-derived CMP-sialic acid (CMP-Neu5Ac in humans). LOS Neu5Ac renders gonococci resistant to complement (by binding the complement inhibitor, factor $\mathrm{H}(\mathrm{FH})$ ) and cationic antimicrobial peptides (CAMPs). LOS sialylation is important for gonococcal virulence in humans and in experimental mouse models. A chimeric protein that fuses the gonococcal binding domains of human FH (lacks complement-inhibiting activity) with human $\operatorname{IgG} 1 \mathrm{Fc}(\mathrm{FH} / \mathrm{Fc})$ enhances complement activation on the bacterium and mediates complement-dependent killing of a wide array of gonococcal isolates in vitro. Intravaginal administration of $\mathrm{FH} / \mathrm{Fc}$ attenuates bacterial burden in the mouse vaginal colonization model. Gonococcal Lst has broad substrate specificity and can utilize CMP salts of sialic acid analogs, such as legionaminic (CMP-Leg) or ketodeoxynonulosonic (CMP-Kdn). Incorporation of Leg or $\mathrm{Kdn}$ into LOS substitutes for the Neu5Ac sialic acid cap and restores bacterial susceptibility to complement and CAMPs. Intravaginal CMP-Leg or CMP-Kdn administration mediates CAMPdependent clearance of MDR Ng in mice. An LOS epitope that is recognized by monoclonal antibody $(\mathrm{mAb}) 2 \mathrm{C} 7$ is expressed by $\sim 95 \%$ of $\mathrm{Ng}$ isolates in vivo, can also be sialylated and is critical for virulence. The 2C7 epitope has also been fashioned as a vaccine candidate and is pending a human trial. mAb $2 \mathrm{C} 7$ delivered either intravaginally or systemically, or as a DNA-encoded mAb (passive vaccination) clears gonococci from mouse vaginas in a complementdependent manner. In conclusion, targeting LOS-related virulence mechanisms is an innovate approach to combat MDR Ng.

\section{PL16 STRENGTHENING COMMUNITIES' RESPONSE, STAKE, AND ENGAGEMENT IN HIV/STI RESEARCH}

S Rawat*. The Humsafar Trust, Mumbai, India

\subsection{6/sextrans-2021-sti.16}

Though HIV interventions have been implemented in India since over a decade, HIV prevalence among men who have sex with men (MSM) and transgender women (TGW) in India $(2.7 \%$ and $3.1 \%$, respectively) continues to be $10-15$ times higher than that observed among heterosexual communities $(0.34 \%)$. While evidence for nationwide prevalence of STIs is lacking, independent studies have estimated STI rates at $12-38 \%$. Further, clinic data from The Humsafar Trust-an LGBTQ+ organization in Mumbai providing HIV outreach services to over $7500 \mathrm{MSM}$ and TGW annuallyhas estimated syphilis rates to be $10 \%$ in 2018 and $12 \%$ in 2019. A key challenge in addressing these STI trends is the limited availability of prevalence-focused programmatic implementation and bio-behavioral research on STI awareness/health seeking behavior among vulnerable communities. Though syphilis testing has been integrated in national HIV interventions, management of other STIs is syndromic resulting in under-diagnoses and under-reporting. Further, lack of dedicated resources toward building knowledge and awareness of STIs among vulnerable communities leads to low risk-perception and compromised uptake of testing and treatment. While evidence building and documentation can 
be strengthened by informed and uniform integration of STI testing within HIV programs, uptake of knowledge, awareness and health services much rely on engagement and involvement of vulnerable communities. Thus, enabling and building community response is critical in addressing issues pertaining to STI management. Community involvement can guide implementation and design for STI-focused interventions; strengthen on-ground outreach; identify barriers; and promote awareness of testing and treatment. As quality evidence is vital for informing strategies, it is critical to strengthen capacities and have communities' involvement in STI research. While community engagement and -stake may not address all challenges, it will be a solid step toward mitigating critical issues of low risk-perception and low uptake of STI testing and treatment among vulnerable communities.

\section{PL17 ENDING THE HIV EPIDEMIC: THE CRITICAL ROLE OF IMPLEMENTATION SCIENCE}

J Smith*. University of Utah School of Medicine, Salt Lake City, UT, USA

10.1136/sextrans-2021-sti. 17

In 2021, the requisite biomedical and behavioral interventions to eliminate new HIV infections exist. 'Ending the HIV Epidemic' worldwide now becomes primarily a challenge of will and implementation. For the past quarter century, the field of implementation science has been developing effective models and strategies to implement effective interventions in real-world service systems with the goal of efficiently providing interventions that reach the right people, at the right time, and in the right place. Yet, successful, sustained implementation is complex and requires a paradigmatic shift to focusing on the upstream factors in the system that are necessary to achieve the clinical effects demonstrated in efficacy research. While HIV/STI researchers have been implementing interventions for years, applying the best available scientific methods to this process has only recently begun to permeate the field and there are a number of gaps in knowledge, training, and application. This plenary will discuss the promising methods from implementation science for Ending the HIV Epidemic and describe some of the efforts in the United States to improve the science and lead to a world with no new HIV infections.

\section{PL18 MINORITY STRESS AND THE HEALTH OF LGBT POPULATIONS}

I Meyer*. UCLA School of Law, Los Angeles, USA

10.1136/sextrans-2021-sti.18

Minority stress theory describes how prejudice and stigma against LGBTQ people leads excess stress-both institutional and interpersonal-and, in turn, to adverse health outcomes. Social changes over the past 70 years in the United States have led to many changes in law and social attitudes toward LGBTQ people. In this presentation I will examine the impact of minority stress historically and today. I will report on results from two innovative U.S. national probability
samples-Generations and TransPop-of lesbian, bisexual, gay, and other sexual minorities (www.generationsstudy.com) and transgender adults (www.transpop.org). These studies cover topics related to identity and coming out, gender transition, exposure to chronic stress, life events, and everyday discrimination, and mental and physical health outcomes. We also examine current level of affiliation of LGBTQ people with the LGBTQ community-something that has been a key feature for delivering services and public health information to LGBTQ people at least since the 1980s. Results show that while we see many positive changes in society and, especially legal protections in the United States, these changes have not resulted in corresponding marked reduction in the experience of minority stress among LGBTQ people and have not reduced markers of poor health such as depression and suicidality. I will discuss the significance of these findings for public policy and future research in LGBTQ populations.

\section{Symposium presentations}

\section{S01.1 IDENTIFICATION OF BACTERIA ASSOCIATED WITH IDIOPATHIC NONGONOCOCCAL URETHRITIS}

S Srinivasan*. Fred Hutchinson Cancer Research Center, Seattle, USA

\subsection{6/sextrans-2021-sti.19}

Nongonococcal urethritis (NGU) is a common syndrome in men with no known etiology in up to $50 \%$ of cases. The most common causes include Chlamydia trachomatis (CT) and Mycoplasma genitalium (MG) accounting for 20\% to $40 \%$ and $10 \%$ to $30 \%$ of NGU cases respectively. Less common causes are Trichomonas vaginalis (TV), herpes simplex virus (HSV) and adenovirus. To identify if other bacteria are associated with NGU, we conducted a large study to estimate associations between urethral bacteria and NGU in men who have sex with men (MSM) and men who have sex with women (MSW). Urine samples were collected from 250 NGU cases and 184 controls attending a Seattle STD clinic. Cases had $\geq 5$ polymorphonuclear leukocytes on Gram stain plus symptoms or discharge; controls had $<5$ PMNs, no symptoms, no discharge. We characterized the urethral microbiota using $16 \mathrm{~S}$ rRNA gene sequencing, used compositional lasso analysis to identify associations between bacterial taxa and NGU and validated the associations with sensitive species-specific quantitative PCR. Among NGU cases, $45.2 \%$ were idiopathic, negative for known causes of NGU including CT, MG, TV, HSV and adenovirus. Our analysis revealed strong associations between detection of Haemophilus influenzae and idiopathic NGU among MSM $(20 \%$ vs. $3 \% ; \mathrm{p}=0.003)$ and MSW $(33 \%$ vs. $1 \% ; \mathrm{p}<0.001)$ while Mycoplasma penetrans was associated with NGU only among MSM $(25 \%$ vs. $1 \% ; \mathrm{p}<0.001)$ and not MSW $(5 \%$ vs. $1 \% ; \mathrm{p}=0.131)$. The two potential new causes of male urethritis, $H$. influenzae and $M$. penetrans, together accounted for an additional $18.8 \%$ of NGU cases in our study. Our current research is aimed at understanding the natural history and treatment outcomes for these bacteria in longitudinal samples that were collected daily and weekly in a subset of participants. Initial observations suggest that $\mathrm{M}$. penetrans can persist for several days post antibiotic therapy. 